\section{Medienbildung in Hauptschul- und Migrationsmilieus}

\section{HORST NIESYTO}

In den letzten Monaten fanden verschiedene Fachtagungen statt, die auf dem Hintergrund der allgemeinen bildungspolitischen Debatte über den prekären Zusammenhang von Schulerfolg und sozialer Herkunft Fragen nach Medienkompetenz und Medienbildung in Hauptschul- und Migrationsmilieus thematisierten:

- Die Landesanstalt für Medien NRW führte am 19. Juni 2006 eine Tagung zum Thema „Förderung von Medienkompetenz. sozial benachteiligter Kinder und Jugendlicher" durch. Gegenstand war die Entwicklung von Handlungsempfehlungen für die Implementierung medienpädagogischer Konzepte für die Arbeit mit jugendlichen Zielgruppen mit besonderem Förderbedarf. Nach einer Kurzpräsentation von vier Modellprojekten in NRW stellten Marion Brüggemann und Jutta Croll Evaluationsergebnisse aus den Projekten vor, ergänzt durch Statements mehrer externer Expertinnen und Experten sowie einer zusammenfassenden Diskussion mit Mitarbeiter/innen der Teilprojekte sowie anwesenden Praktiker/innen aus der Medienarbeit.

Anbei das Experten-Statement von Prof. Dr. Horst Niesyto (Abteilung Medienpädagogik, PH Ludwigsburg).

Die zusammenfassenden Handlungsempfehlungen werden im Laufe der nächsten Monate von der Landesanstalt für Medien NRW veröffentlicht werden.

- „Schulen ans Netz“ führte am 20. September 2006 eine Fachtagung in Bonn zum Thema „Lernen, Migration und digitale Medien - Potenziale für Jugendliche mit Migrationshintergrund Hauptschülerinnen und Hauptschüler)" durch. Vorgestellt wurde eine neue Internetplattform „LIFT“ - Lernen, Integrieren, Fördern und Trainieren (webbasierte Lernangebote für Jugendliche mit Migrationshintergrund), ergänzt durch drei Fachvorträge und eine Podiumsdiskussion.

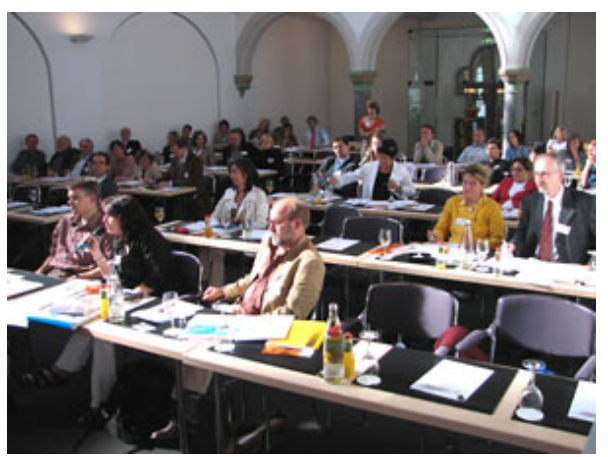

Prof'in Dr. Annette Treibel (PH Karlsruhe) referierte zum Thema „Lebenssituation und Mediennutzung von Hauptschülerinnen und Hauptschülern mit Migrationshintergrund":

Unter welchen sozio-ökonomischen Gegebenheiten wachsen Jugendliche mit Migrationshintergrund auf? Wie nutzen sie die digitalen Medien und welche Bedeutung haben sie für ihre kulturelle Identität?

Annette Treibel informierte vor allem über Ergebnisse einer empirischen Studie zur Mediennutzung türkischer und russischer Jugendlicher,

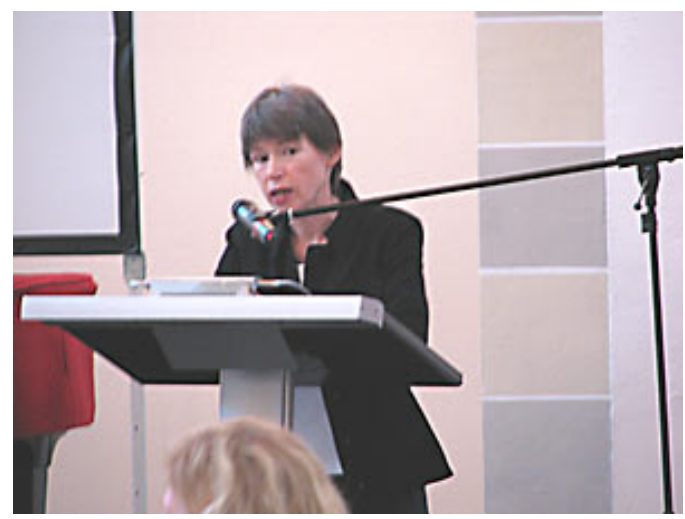

die in Deutschland leben. Als ein Trend wurde deutlich, dass Medienwissen im Sinne von Bedienungs- und Handlungswissen nur bedingt vorhanden ist. Im Unterschied zu Peergroup und Schule kommt den familiären Sozialisationsprozessen eine prägende Bedeutung zu. Im Spannungsfeld von elterlicher Aufmerksamkeit und Kontrolle könne nicht gesagt werden, dass „Familie“ (als soziale Herkunft) generell ein „bildungshemmender“ Faktor sei - Familienzusammenhänge erscheinen vielmehr als eine wichtige Ressource für die Orientierung dieser Jugendlichen, da Schule und Gesellschaft als „nicht verlässlich“ betrachtet werden.

Jun.-Prof. Dr. Michael Klebl (Fernuniversität Hagen) legte in seinem Vortrag „Fremd und Eigen: Webbasierte Lernangebote zwischen strukturierten Lernprozessen und informellem Lernen" einen Schwerpunkt auf lerntheoretische und mediendidaktische Aspekte:

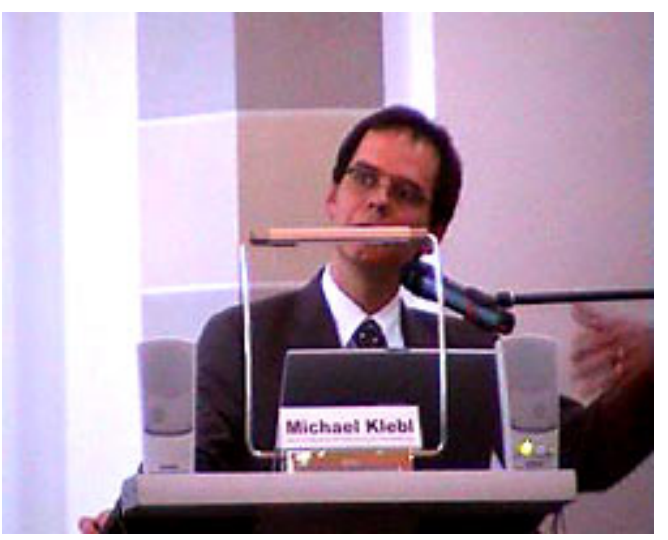


Wie lernen bildungsbenachteiligte Jugendliche und insbesondere Jugendliche mit Migrationshintergrund mit digitalen Medien (Lernformen, Lernszenarien etc.)? Wie können digitale Medien Lern- und Bildungsprozesse nachhaltig unterstützen?

Michael Klebl stellte verschiedene Dimensionen eines sozial-konstruktivistischen Lernkonzepts vor, welche das Erlernen von Sprachen in (soziale) Handlungsbezüge einbettet. Ausgehend von der Reflexion unterschiedlicher Modelle des Spracherwerbs, die aktuell in der Diskussion sind (erst eigene Sprache, dann Fremdsprache versus bilingualer Spracherwerb) skizzierte M. Klebl verschiedene Kriterien für internetbasierte Lernszenarien. Er akzentuierte die Notwendigkeit sowohl lernzielorientierter Planung von Lernprozessen in formellen Kontexten (bei Gewährleistung von Transparenz der einzelnen Schritte für die Lernenden) als auch neue, informelle Lernformen in Netzwerken (learning communities: virtuelle Öffentlichkeiten von Migranten im Netz).

Prof. Dr. Horst Niesyto (PH Ludwigsburg) referierte zum Thema „Interkulturelle Medienpädagogik - Anforderungen an (digitale) Medienangebote für Jugendliche aus Hauptschul-und Migrationsmilieus":

Welche Aufgabe hat interkulturelle Medienbildung? Wie sollten Medienangebote konzipiert sein, um Jugendliche aus sog. bildungsfernen Milieus erreichen zu können?

Horst Niesyto gab zunächst einen Überblick über wesentliche Ziele und Aufgaben interkultureller Medienpädagogik und arbeitete gemeinsame Zielsetzungen von interkultureller Pädagogik und interkultureller Medienbildung heraus. Im Mittelpunkt seines Vortrags standen

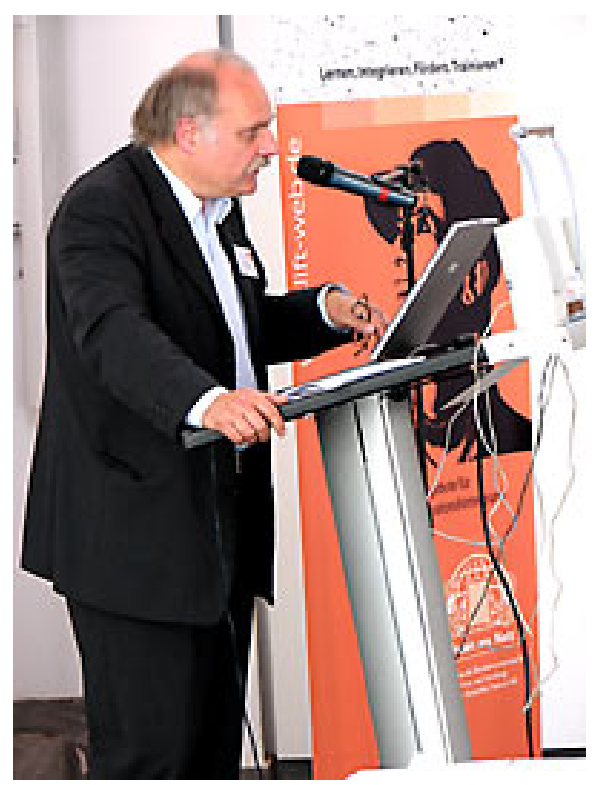

Erfahrungswerte aus verschiedenen medienpädagogischen Modellprojekten, insbesondere aus dem Projekt „CHICAM - Children in Communication about Migration“. Niesyto betonte, dass eine einseitige Orientierung auf „systematischen Schriftspracherwerb“ nicht ausreicht, um die Integrationschancen von Kindern und Jugendlichen aus Hauptschul- und Migrationsmilieus zu verbessern. Notwendig ist ein integriertes Konzept einer umfassenden „Alphabetisierung“, das wort- und schriftsprachliche mit bildhaften und multimedialen Ausdrucks- und Kommunikationsformen in eine Balance bringt und Verknüpfungen herstellt. Erfahrungen aus Projekten zeigen, dass lebensweltbezogene, anschauliche, spielerische und assoziativ-intuitive Arbeitsformen besonders geeignet sind, die Motivation von Jugendlichen zu fördern. Gleichzeitig braucht es einer gewissen Struktur, die den jeweiligen Wissensbeständen, Lernvoraussetzungen und Bedürfniskonstellationen gerecht wird - sowohl für das Erstellen der Medienproduktionen als auch für die Nutzung von Internet-Plattformen.

In der abschließenden Podiumsdiskussion über „Perspektiven für das Lernen mit digitalen $\mathrm{Me}$ dien von Jugendlichen mit Migrationshintergrund" ging es u.a. um die Anforderungen für das Lernen mit digitalen Medien und um Erfahrungswerte aus einzelnen Praxisfeldern und Projekten in verschiedenen Bundesländern.

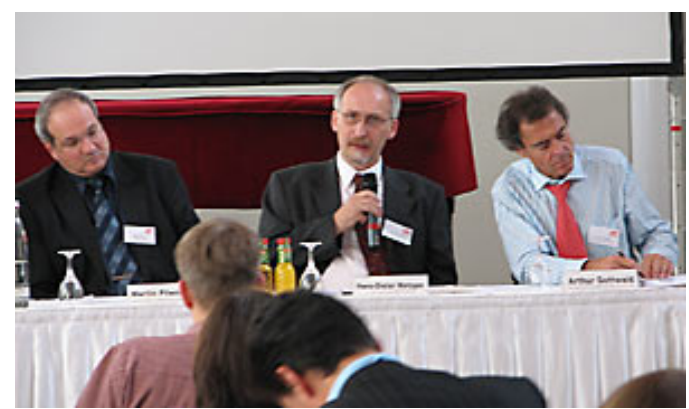

v.l.n.r.: Dr. Martin Plieninger (PH SchwäbischGmünd), Dr. Hans-Dieter Metzger (Schulreferat Nürnberg), Arthur Gottwald (Behörde für Bildung und Sport Hamburg)

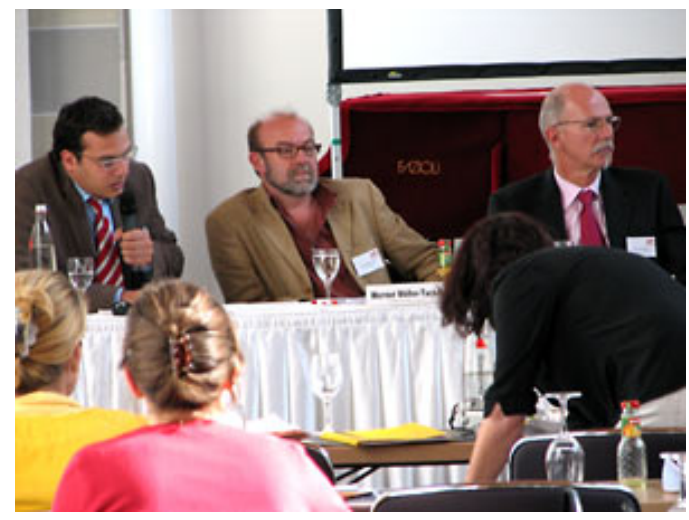

v.l.n.r.: Birand Bingül (Moderator), Werner Möller-Tacke (Modellprogramm „Förderung von Kindern und Jugendlichen mit Migrationshintergrund“, NRW), Reinhard Schwalbach (IJAB Bonn). 
Auch dieser Veranstaltungsteil unterstrich die Notwendigkeit, deutlicher als bislang von den vorhandenen Stärken und Ausdruckspotenzialen von Jugendlichen mit Migrationshintergrund auszugehen und eine „Defizitperspektive" zu überwinden. Als sinnvoll erscheinen Lernumgebungen, die sowohl strukturierte Aufgaben und Übungen enthalten als auch genügend Räume für eigenes Gestalten, Ausprobieren und Kommunizieren bieten. In diesem Sinne darf man gespannt sein, wie sich die LIFT-Lernplattform entwickeln wird.

Literaturhinweise zur Thematik (Downloads im Netz)

Internationale Fachtagung: Grenzenlose Cyberwelt? Digitale Ungleichheit und neue Bildungszugänge für Jugendliche. Internationale Fachtagung, 09.-11. Februar 2006, Bielefeld.

H. Niesyto: Chancen und Perspektiven interkultureller Medienpädagogik. 